\title{
Cooper pairing and BCS-BEC evolution in mixed-dimensional Fermi gases
}

\author{
M. Iskin \\ Department of Physics, Koç University, Rumelifeneri Yolu, 34450 Sariyer, Istanbul, Turkey \\ A. L. Subaş1* \\ Faculty of Engineering and Natural Sciences, Sabanci University, 34956 Tuzla, Istanbul, Turkey
}

(Received 21 April 2010; published 27 December 2010)

\begin{abstract}
Similar to what has recently been achieved with Bose-Bose mixtures [G. Lamporesi, J. Catani, G. Barontini, Y. Nishida, M. Inguscio, and F. Minardi, Phys. Rev. Lett. 104, 153202 (2010)], mixed-dimensional Fermi-Fermi mixtures can be created by applying a species-selective one-dimensional optical lattice to a two-species Fermi gas $(\sigma \equiv\{\uparrow, \downarrow\})$, in such a way that both species are confined to quasi-two-dimensional geometries determined by their hoppings along the lattice direction. We investigated the ground-state phase diagram of superfluidity for such mixtures in the BCS-BEC evolution, and found normal, gapped superfluid, gapless superfluid, and phase separated regions. In particular, we found a stable gapless superfluid phase where the unpaired $\uparrow$ and $\downarrow$ fermions coexist with the paired (or superfluid) ones in different momentum space regions. This phase is in some ways similar to the Sarma state found in mixtures with densities, but in our case, the gapless superfluid phase is unpolarized and most importantly it is stable against phase separation.
\end{abstract}

DOI: 10.1103/PhysRevA.82.063628

PACS number(s): $03.75 . S s$

\section{INTRODUCTION}

Atomic Fermi gases have emerged as a unique testing ground for many theories of exotic matter in nature, allowing for the creation of complex yet very controllable many-body quantum systems, [1] where for instance observation of the BCS-BEC crossover has so far been the most important achievement in this field. Following this huge success with single-species fermion mixtures, there has been increasing experimental interest in studying two-species Fermi-Fermi mixtures [2-7]. In particular, ${ }^{6} \mathrm{Li}^{40}{ }^{40} \mathrm{~K}$ mixtures have recently been trapped and interspecies Feshbach resonances have been identified, opening a new frontier in ultracold atomic research to study exotic many-body phenomena, one of which is the possibility of studying fermion pairing in mixed dimensions [8,9].

Mixed-dimensional atomic systems, in which two types of particles live in different dimensions, can be created with two-species Fermi-Fermi, Bose-Fermi, and Bose-Bose mixtures by using species-selective optical lattice potentials. This has recently been achieved with Bose-Bose mixtures [10] by applying a one-dimensional optical lattice to a ${ }^{41} \mathrm{~K}-{ }^{87} \mathrm{Rb}$ mixture, where only ${ }^{41} \mathrm{~K}$ atoms feel the lattice potential and are confined to a quasi-two-dimensional geometry, while having negligible effect on ${ }^{87} \mathrm{Rb}$ atoms, thus leaving the ${ }^{87} \mathrm{Rb}$ atoms three dimensional. Motivated by this experimental work, here we analyze Cooper pairing in mixed-dimensional Fermi gases. We consider both single-species and two-species fermion mixtures, and analyze the ground state phase diagrams in the BCS-BEC evolution, which involves normal, gapped superfluid, gapless superfluid, and phase separated regions. In particular, the gapless superfluid phase, where the unpaired $\uparrow$ and $\downarrow$ fermions coexist with the paired (or superfluid) ones in different momentum space regions, is in some ways similar to the Sarma state found in mixtures with unequal densities [11],

\footnotetext{
*Current address: Department of physics, Faculty of Science and Letters, Istanbul Technical University, 34469 Maslak, Istanbul, Turkey.
}

but in our case, the gapless superfluid phase is unpolarized and most importantly it is stable against phase separation. In this way, our gapless superfluid phase is very similar to those of Refs. [12,13] which are recently proposed for ultracold atomic systems in other contexts.

The rest of this article is organized as follows. In Sec. II, after introducing the Hamiltonian in Sec. II A, the corresponding saddle-point self-consistency equations are derived in Sec. II B, and their noninteracting limit is discussed in Sec. IIC. We numerically solve these equations in the BCS-BEC evolution in Sec. III where we investigate the normal-superfluid transition in Sec. III A the topological gapless-gapped superfluidity transition in Sec. III B and the ground state phase diagrams in Sec. III C. A brief summary of our conclusions is given in Sec. IV. The details of the self-consistency equations at $T=0$ are further discussed in Appendix A and the boundary equation for the normalsuperfluid transition is derived in Appendix B.

\section{MIXED-DIMENSIONAL FERMI GASES}

In this work, we analyze Cooper pairing in mixeddimensional Fermi gases, which seems to be a very promising way to create superfluidity with mismatched Fermi surfaces, and the physics involved is in some ways similar to that of unequal density mixtures [14-17]. We consider only uniform (homogenous) mixtures, but emphasize that the finite-size effects due to the confining trapping potentials (which are always present in atomic systems) can be taken into account using the local-density approximation (as a first approximation).

\section{A. Hamiltonian}

To describe such mixed-dimensional Fermi gases in a species-selective one-dimensional optical lattice (say in the $\widehat{\mathbf{z}}$ direction), we start with the real-space Hamiltonian 


$$
\begin{aligned}
\left(\hbar=k_{B}=\right. & 1) \\
H= & \sum_{\sigma} \int d^{3} \mathbf{r} \psi_{\mathbf{r}, \sigma}^{\dagger}\left[-\frac{\nabla^{2}}{2 m_{\sigma}}-\mu_{\sigma}+V_{\mathrm{OL}}^{\sigma}\left(r_{z}\right)\right] \psi_{\mathbf{r}, \sigma} \\
& -g \int d^{3} \mathbf{r} \psi_{\mathbf{r}, \uparrow}^{\dagger} \psi_{\mathbf{r}, \downarrow}^{\dagger} \psi_{\mathbf{r}, \downarrow} \psi_{\mathbf{r}, \uparrow},
\end{aligned}
$$

where the pseudospin $\sigma$ labels both the type and hyperfine states of atoms represented by the creation operator $\psi_{\mathbf{r}, \sigma}^{\dagger}, m_{\sigma}$ is the mass, and $\mu_{\sigma}$ is the chemical potential. Here, $\mathbf{r} \equiv\left(r_{x}, r_{y}, r_{z}\right)$ is the position with $r_{\perp}=\sqrt{r_{x}^{2}+r_{y}^{2}}, V_{\mathrm{OL}}^{\sigma}=V_{0, \sigma} \sin ^{2}\left(\pi r_{z} / d_{z}\right)$ is the optical lattice potential, $d_{z}$ is the lattice spacing, and $g \geqslant 0$ is the strength of the attractive interaction (zero-range and isotropic) between $\uparrow$ and $\downarrow$ fermions.

In order to obtain the momentum-space Hamiltonian, we first expand the creation and annihilation field operators in the orthonormal and complete basis set of Wannier functions $W\left(r_{z}-r_{z}^{i}\right)$ of the lowest energy states of the optical potential near their minima (single-band approximation), and then take the Fourier transform of the site operators. This corresponds to the following substitution: $\psi_{\mathbf{r}, \sigma}=(1 / \sqrt{M}) \sum_{i, \mathbf{k}} a_{\mathbf{k}, \sigma} W_{\sigma}\left(r_{z}-\right.$ $\left.r_{z}^{i}\right) e^{-i\left(\mathbf{k}_{\perp} \cdot \mathbf{r}_{\perp}+k_{z} r_{z}^{i}\right)}$, where $\mathbf{k} \equiv\left(k_{x}, k_{y}, k_{z}\right)$ is the momentum with $k_{\perp}=\sqrt{k_{x}^{2}+k_{y}^{2}}, i$ labels the lattice sites, and $M$ is the total number of them. Keeping only the tunneling between nearest neighbor sites and onsite interactions, and using the orthonormality of the Wannier functions, the resultant Hamiltonian can be written as

$$
H=\sum_{\mathbf{k}, \sigma} \xi_{\mathbf{k}, \sigma} a_{\mathbf{k}, \sigma}^{\dagger} a_{\mathbf{k}, \sigma}-g \sum_{\mathbf{k}, \mathbf{k}^{\prime}, \mathbf{q}} b_{\mathbf{k}, \mathbf{q}}^{\dagger} b_{\mathbf{k}^{\prime}, \mathbf{q}}
$$

where $\quad b_{\mathbf{k}, \mathbf{q}}^{\dagger}=a_{\mathbf{k}+\mathbf{q} / 2, \uparrow}^{\dagger} a_{-\mathbf{k}+\mathbf{q} / 2, \downarrow}^{\dagger}$ creates fermion pairs with center of mass momentum $\mathbf{q}$ and relative momentum 2k. Here, $\xi_{\mathbf{k}, \sigma}=\epsilon_{\mathbf{k}, \sigma}-\mu_{\sigma}$, where $\epsilon_{\mathbf{k}, \sigma}=$ $k_{\perp}^{2} /\left(2 m_{\sigma}\right)+2 t_{\sigma}\left[1-\cos \left(k_{z} d_{z}\right)\right]$ is the single-particle energy dispersion, and $t_{\sigma}=\int d r_{z} W^{*}\left(r_{z}-r_{z}^{i}\right)\left[-\partial^{2} /\left(2 m_{\sigma} \partial r_{z}^{2}\right)+\right.$ $\left.V_{\mathrm{OL}}^{\sigma}\left(r_{z}\right)\right] W\left(r_{z}-r_{z}^{j}\right)$ is the tunneling amplitude between any nearest neighbor sites $i$ and $j$.

Following the usual treatment, the strength of the attractive interaction can be written in terms of an "effective" $s$ wave scattering length $a_{\mathrm{eff}}[18]$ as $1 / g=-m_{+} V /\left(4 \pi a_{\mathrm{eff}}\right)+$ $\sum_{\mathbf{k}} 1 /\left(2 \epsilon_{\mathbf{k},+}\right)$, where $m_{ \pm}=2 m_{\uparrow} m_{\downarrow} /\left(m_{\downarrow} \pm m_{\uparrow}\right), \quad V$ is the volume of the system, and $\epsilon_{\mathbf{k}, \pm}=\left(\epsilon_{\mathbf{k}, \uparrow} \pm \epsilon_{\mathbf{k}, \downarrow}\right) / 2$. Note that $m_{+}$is twice the reduced mass of the $\uparrow$ and $\downarrow$ fermions, and that the equal mass case corresponds to $\left|m_{-}\right| \rightarrow \infty$. Here, and throughout, the momentum space sums are evaluated as $\sum_{\mathbf{k}} \equiv\left[V /(2 \pi)^{3}\right] \int d^{3} \mathbf{k} \equiv\left[V /\left(2 \pi^{2}\right)\right] \int_{0}^{\pi / d_{z}} d k_{z} \int_{0}^{\infty} k_{\perp} d k_{\perp}$, since the system at hand has a cylindrical symmetry around the $k_{z}$ axis, and a translational symmetry along the $\widehat{\mathbf{z}}$ direction, so that the $k_{z}$ integrals are limited to the first Brillouin zone, i.e., $-\pi / d_{z} \leqslant k_{z} \leqslant \pi / d_{z}$. The resultant integrands are also even functions of $k_{z}$, and hence we integrate over half of the Brillouin zone and multiply them by two.

In this article, for simplicity, we set $t_{\downarrow}=1 /\left(2 m_{\downarrow} d_{z}^{2}\right)$ but allow for the $\uparrow$ fermions to have different effective masses along the lattice direction through a tight-binding dispersion determined by $t_{\uparrow}$, so that

$$
\begin{gathered}
\epsilon_{\mathbf{k}, \uparrow}=\frac{k_{\perp}^{2}}{2 m_{\uparrow}}+2 t_{\uparrow}\left[1-\cos \left(k_{z} d_{z}\right)\right], \\
\epsilon_{\mathbf{k}, \downarrow}=\frac{k_{\perp}^{2}}{2 m_{\downarrow}}+\frac{1}{m_{\downarrow} d_{z}^{2}}\left[1-\cos \left(k_{z} d_{z}\right)\right] .
\end{gathered}
$$

Notice that when $k_{F z, \downarrow} d_{z} \ll \pi$ (i.e., the low-filling limit for the $\downarrow$ species), where $k_{F z} \downarrow$ is the Fermi momentum of $\downarrow$ fermions in the $k_{z}$ direction, Eq. (4) can be approximated as $\epsilon_{\mathbf{k}, \downarrow} \approx k^{2} /\left(2 m_{\downarrow}\right)$. Next, we analyze the phase diagram of the Hamiltonian given in Eq. (2) with the dispersions given by Eqs. (3) and (4), within the saddle-point (mean-field) approximation.

\section{B. Self-consistency equations}

At low temperatures $(T \approx 0)$, the saddle-point selfconsistency (order parameter and number) equations are sufficient to describe the BCS-BEC evolution of superfluidity $[19,20]$. For the Hamiltonian given in Eq. (2) the saddle-point action is $S_{0}=\Omega_{0} / T$, where

$$
\Omega_{0}=\frac{|\Delta|^{2}}{g}+\sum_{\mathbf{k}}\left(\xi_{\mathbf{k},+}-E_{\mathbf{k},+}\right)+T \sum_{\mathbf{k}, s} \ln \left[f\left(-E_{\mathbf{k}, s}\right)\right]
$$

is the saddle-point thermodynamic potential. Here, $f(x)=$ $1 /[\exp (x / T)+1]$ is the Fermi function, $E_{\mathbf{k}, s}=\left(\xi_{\mathbf{k},+}^{2}+\right.$ $\left.|\Delta|^{2}\right)^{1 / 2}+\gamma_{s} \xi_{\mathbf{k},-}$ is the quasiparticle energy when $\gamma_{1}=1$ or the negative of the quasihole energy when $\gamma_{2}=-1$, and $E_{\mathbf{k}, \pm}=\left(E_{\mathbf{k}, 1} \pm E_{\mathbf{k}, 2}\right) / 2$. In addition, $\Delta$ is the order parameter and $\xi_{\mathbf{k}, \pm}=\epsilon_{\mathbf{k}, \pm}-\mu_{ \pm}$, where $\mu_{ \pm}=\left(\mu_{\uparrow} \pm \mu_{\downarrow}\right) / 2$. Note that the symmetry between quasiparticles and quasiholes is broken when $\xi_{\mathbf{k},-} \neq 0$.

The saddle-point condition $\delta \Omega_{0} / \delta \Delta^{*}=0$ leads to an equation for the order parameter,

$$
-\frac{m_{+} V}{4 \pi a_{\mathrm{eff}}}=\sum_{\mathbf{k}}\left[\frac{1-f\left(E_{\mathbf{k}, 1}\right)-f\left(E_{\mathbf{k}, 2}\right)}{2 E_{\mathbf{k},+}}-\frac{1}{2 \epsilon_{\mathbf{k},+}}\right],
$$

where, as usual, $g$ is eliminated in favor of the effective $s$-wave scattering length $a_{\text {eff }}$ via the relation given above $[19,20]$. The order parameter equation has to be solved self-consistently with the number equations. At the saddle point, the relation $N_{\sigma}=-\partial \Omega_{0} / \partial \mu_{\sigma}$ leads to

$$
\begin{aligned}
& N_{\uparrow}=\sum_{\mathbf{k}}\left[\left|u_{\mathbf{k}}\right|^{2} f\left(E_{\mathbf{k}, 1}\right)+\left|v_{\mathbf{k}}\right|^{2} f\left(-E_{\mathbf{k}, 2}\right)\right], \\
& N_{\downarrow}=\sum_{\mathbf{k}}\left[\left|u_{\mathbf{k}}\right|^{2} f\left(E_{\mathbf{k}, 2}\right)+\left|v_{\mathbf{k}}\right|^{2} f\left(-E_{\mathbf{k}, 1}\right)\right],
\end{aligned}
$$

where $\quad\left|u_{\mathbf{k}}\right|^{2}=\left(1+\xi_{\mathbf{k},+} / E_{\mathbf{k},+}\right) / 2 \quad$ and $\quad\left|v_{\mathbf{k}}\right|^{2}=(1-$ $\left.\xi_{\mathbf{k},+} / E_{\mathbf{k},+}\right) / 2$ are the usual coherence factors. At $T=0$, Eqs. (6), (7), and (8) can be simplified considerably as shown in Appendix A.

In order to analyze the phase diagram at $T=0$, we solve the saddle-point self-consistency equations and check the stability of these solutions for the uniform superfluid phase using the compressibility (or the curvature) criterion [21-23]. This says that the compressibility matrix $\kappa(T)$ with elements $\kappa_{\sigma, \sigma^{\prime}}(T)=-\partial^{2} \Omega_{0} /\left(\partial \mu_{\sigma} \partial \mu_{\sigma^{\prime}}\right)$ needs to be positive definite, 
and it is related (identical) to the condition that the curvature

$$
\frac{\partial^{2} \Omega_{0}}{\partial \Delta^{2}}=|\Delta|^{2} \sum_{\mathbf{k}, s}\left[\frac{0.5-f\left(E_{\mathbf{k}, s}\right)}{E_{\mathbf{k},+}^{3}}+\frac{f^{\prime}\left(E_{\mathbf{k}, s}\right)}{E_{\mathbf{k},+}^{2}}\right]
$$

of the saddle-point thermodynamic potential $\Omega_{0}$ with respect to the saddle-point parameter $\Delta$ needs to be positive. Here, $f^{\prime}(x)=d f(x) / d x$. When at least one of the eigenvalues of $\kappa(T)$, or the curvature $\partial^{2} \Omega_{0} / \partial \Delta^{2}$, is negative, the uniform saddle-point solution does not correspond to a minimum of $\Omega_{0}$, and a nonuniform superfluid phase, e.g., a phase separation, is favored.

\section{Noninteracting limit}

Before presenting our numerical results, let us first analyze the noninteracting $g \rightarrow 0$ limit. In this limit, since $1 / a_{\mathrm{eff}} \rightarrow$ $-\infty$, the order parameter vanishes $\Delta \rightarrow 0$, and at $T=0$ Eqs. (7) and (8) can be written as $N_{\sigma}=\sum_{\mathbf{k}} \theta\left(-\xi_{\mathbf{k}, \sigma}\right)$, where $\theta(x)$ is the Heaviside step function. Evaluating the $\mathbf{k}$-space sums, the density $n_{\sigma}=N_{\sigma} / V$ of the $\sigma$ fermions becomes

$$
\begin{gathered}
n_{\sigma}=\frac{m_{\sigma} k_{F z, \sigma}}{2 \pi^{2}}\left(\mu_{\sigma}-2 t_{\sigma}\right)+\frac{m_{\sigma} t_{\sigma}}{\pi^{2} d_{z}} \sin \left(k_{F z, \sigma} d_{z}\right), \\
\mu_{\sigma}=2 t_{\sigma}\left[1-\cos \left(k_{F z, \sigma} d_{z}\right)\right],
\end{gathered}
$$

where $k_{F z, \sigma} \geqslant 0$ corresponds to the Fermi momentum of the $\sigma$ fermions in the $k_{z}$ direction, which is related to the chemical potential via Eq. (11).

Note that, in the low-density $\left(k_{F z, \downarrow} d_{z} \ll \pi\right)$ limit, expanding out Eqs. (10) and (11) to the lowest nontrivial orders (third and second orders, respectively) in $k_{F z, \downarrow} d_{z}$, we obtain $n_{\downarrow} \approx m_{\downarrow} k_{F z, \downarrow}\left(\mu_{\downarrow}-t_{\downarrow} k_{F z, \downarrow}^{3} d_{z}^{2} / 3\right) /\left(2 \pi^{2}\right)$ and $\mu_{\downarrow} \approx t_{\downarrow} k_{F z, \downarrow}^{2} d_{z}^{2}$. Combining these two expressions, and setting $k_{F z, \downarrow}=k_{F, \downarrow}$ or $t_{\downarrow}=1 /\left(2 m_{\downarrow} d_{z}^{2}\right)$, the density of $\downarrow$ fermions acquires the usual form $n_{\downarrow} \approx k_{F, \downarrow}^{3} /\left(6 \pi^{2}\right)$. In addition, when $t_{\uparrow} \rightarrow 0$ and $k_{F z, \uparrow}=\pi / d_{z}$, Eq. (10) reduces to the density of $\uparrow$ fermions in each (isolated) two-dimensional plane along the $k_{z}$ direction, $N_{\uparrow} /(M A)=m_{\uparrow} k_{F, \uparrow}^{2} /(4 \pi)$, which is of the usual form, where we used $V=A L_{z}$ with $A$ being the area of the system in the $(x, y)$ plane and $L_{z}$ being the system size in the $\widehat{\mathbf{z}}$ direction. Here, $M=L_{z} / d_{z}$ is the number of two-dimensional planes, i.e., the number of lattice sites, in the $\widehat{\mathbf{z}}$ direction. Having discussed the noninteracting limit, next we analyze the BCS-BEC evolution.

\section{SADDLE-POINT APPROXIMATION}

In this section, we consider only equal density mixtures, where $n_{\uparrow}=n_{\downarrow}$, at zero temperature. For these mixtures, first we analyze the amplitude of the order parameter $|\Delta|$, the chemical potential sum $\mu_{+}$, and the chemical potential difference $\mu_{-}$as a function of the tunneling amplitude $t_{\uparrow}$ and effective scattering length $1 /\left(k_{F, \downarrow} a_{\text {eff }}\right)$ for fixed values of $k_{F, \downarrow} d_{z}$. Here, $k_{F, \downarrow}$ is an "effective" Fermi momentum for $\downarrow$ fermions defined through the three-dimensional density $n_{\downarrow}=k_{F, \downarrow}^{3} /\left(6 \pi^{2}\right)$, where $n_{\downarrow}$ is given by Eq. (10). Then, using the stability criterion given in Eq. (9), we construct the phase diagrams. For simplicity, we mainly present our results for the equal mass $\left(m_{\uparrow}=m_{\downarrow}\right)$ mixtures, but we also briefly mention the effects of mass anisotropy $m_{\uparrow} \neq m_{\downarrow}$ on the phase diagrams.

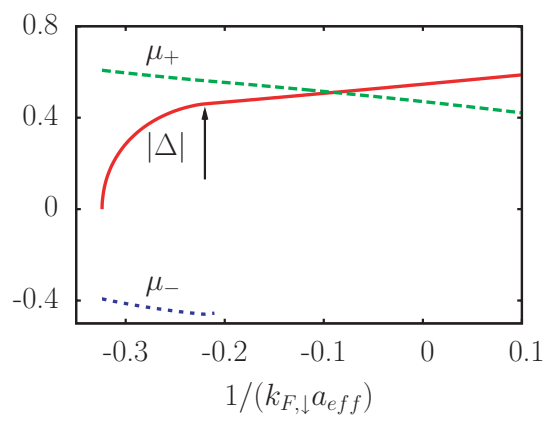

FIG. 1. (Color online) Saddle-point solutions for the amplitude of the order parameter $|\Delta|$, the chemical potential sum $\mu_{+}=\left(\mu_{\uparrow}+\right.$ $\left.\mu_{\downarrow}\right) / 2$, and the chemical potential difference $\mu_{-}=\left(\mu_{\uparrow}-\mu_{\downarrow}\right) / 2$ shown (in units of $\epsilon_{F, \downarrow}$ ) as functions of the effective $s$-wave scattering parameter $1 /\left(k_{F, \downarrow} a_{\mathrm{eff}}\right)$. ( $\mu_{-}$is shown only for weak interactions where it is relevant). These data correspond to the case where $m_{\uparrow}=m_{\downarrow}, t_{\uparrow}=\epsilon_{F, \downarrow}$, and $k_{F, \downarrow} d_{z}=0.1$. The arrow shows the location of the topological gapless-superfluid-to-gapped-superfluid transition discussed in the text.

\section{A. Normal-superfluid transition}

Using $k_{F, \downarrow}$ and $\epsilon_{F, \downarrow}=k_{F, \downarrow}^{2} /\left(2 m_{\downarrow}\right)$ as our length and energy scales, respectively, we solve Eqs. (A1), (A2) and (A3) numerically. For instance, in Fig. 1 we show self-consistent solutions of $|\Delta|, \mu_{+}$, and $\mu_{-}$as functions of $1 /\left(k_{F, \downarrow} a_{\text {eff }}\right)$, when $m_{\uparrow}=m_{\downarrow}, t_{\uparrow}=\epsilon_{F, \downarrow}$, and $k_{F, \downarrow} d_{z}=0.1$. When the scattering parameter is smaller than a critical value, i.e., $1 /\left(k_{F, \downarrow} a_{\text {eff }}\right) \lesssim$ -0.32 , the saddle-point solution is $\mu_{\downarrow}=\mu_{+}-\mu_{-} \rightarrow 1$ (in units of $\left.\epsilon_{F, \downarrow}\right)$ since $|\Delta|=0$, indicating that the mixture is a normal Fermi gas. Beyond this critical value, the superfluid order parameter $|\Delta|$ becomes nonzero, indicating a quantum phase transition from the normal to a superfluid phase.

This transition can be understood from earlier works on Cooper pairing with mismatched Fermi surfaces. For instance, in the case of Fermi gases with unequal densities in purely three dimensions, a superfluid-to-normal phase transition has been recently observed beyond a critical density difference depending on the value of the scattering parameter [14-17]. This transition occurs when the difference in the chemical potentials (or Fermi momenta) reaches what is known as the Clogston-Chandrasekhar limit [24,25]. In our case, the main mechanism is the same. As can be extracted from Eq. (11), the mismatch is inevitable in some parts of the $\mathbf{k}$ space even for the equal density mixtures considered in this analysis. Therefore, it is energetically more favorable for the mixture to be in the normal phase until a critical scattering parameter is reached, beyond which Cooper pairing is possible.

\section{B. Topological gapless-gapped superfluidity transition}

With further increase in the scattering parameter, $|\Delta|$ increases quite rapidly and $\mu_{+}$decreases, with a kink in the former quantity at $1 /\left(k_{F, \downarrow} a_{\text {eff }}\right) \approx-0.22$. (We also expect a weak kink in $\mu_{+}$at the same point, but it is not clearly seen in the data.) Therefore, the BCS-BEC evolution in mixed-dimensional Fermi gases is nonanalytic, i.e., it is not a crossover. Recall that, in usual three-dimensional mixtures, the evolution of $|\Delta|$ and $\mu_{+}$is analytic for all $1 /\left(k_{F, \downarrow} a_{\mathrm{eff}}\right)$, 
(a)

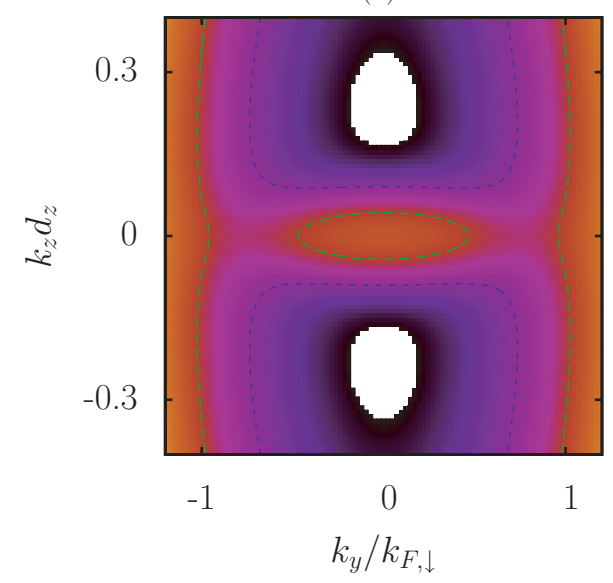

(b)

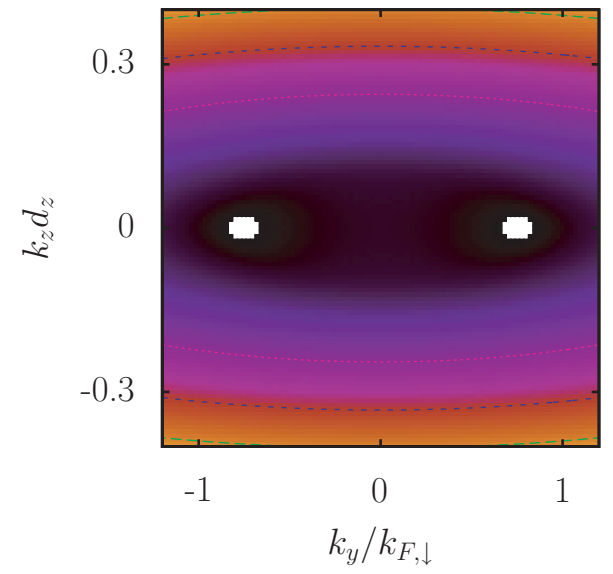

FIG. 2. (Color online) Contour maps of (a) the quasiparticle excitation energy $E_{\mathbf{k}, 1}$, and (b) the negative of the quasihole excitation energy $E_{\mathbf{k}, 2}$, shown as a function of momentum components $k_{z}$ (in units of $1 / d_{z}$ ) and $k_{y}$ (in units of $k_{F, \downarrow}$ ) in the $k_{x}=0$ plane. These data correspond to the case where $m_{\uparrow}=m_{\downarrow}, t_{\uparrow}=\epsilon_{F, \downarrow}, k_{F, \downarrow} d_{z}=0.1$, and $1 /\left(k_{F, \downarrow} a_{\mathrm{eff}}\right)=-0.25$. The excitations are gapless $\left(E_{\mathbf{k}, s} \leqslant 0\right)$ at the white region boundaries.

and the evolution is just a crossover. The kink in $|\Delta|$ is more pronounced for lower values of $t_{\uparrow}$, and it signals a topological quantum phase transition as discussed next.

The excitation spectrum of quasiparticles is determined by energies $E_{\mathbf{k}, 1}$ and $E_{\mathbf{k}, 2}$. At $\mathbf{k}$-space points, the condition $E_{\mathbf{k}, s}=$ 0 defines Fermi surfaces of quasiparticles in momentum space where the quasiparticle excitation spectrum changes from a gapped to a gapless phase. These changes in the Fermi surfaces of quasiparticles are topological in nature [26], and we identify topological quantum phase transitions associated with the disappearance or appearance of momentum space regions of zero quasiparticle energies when either $1 /\left(k_{F, \downarrow} a_{\mathrm{eff}}\right), t_{\uparrow}$, and/or $k_{F, \downarrow} d_{z}$ is changed. Note that the topological transition occurs without changing the symmetry of the order parameter as the Landau classification demands for ordinary phase transitions.

We illustrate the gapless superfluid phase in Fig. 2, where contour maps of $E_{\mathbf{k}, s}$ are shown as functions of $k_{z}$ and $k_{y}$ in the $k_{x}=0$ plane, when $m_{\uparrow}=m_{\downarrow}, t_{\uparrow}=\epsilon_{F, \downarrow}, k_{F, \downarrow} d_{z}=0.1$, and $1 /\left(k_{F, \downarrow} a_{\text {eff }}\right)=-0.25$. In Fig. 1 , these data correspond to a point that is slightly on the left-hand side of the transition point
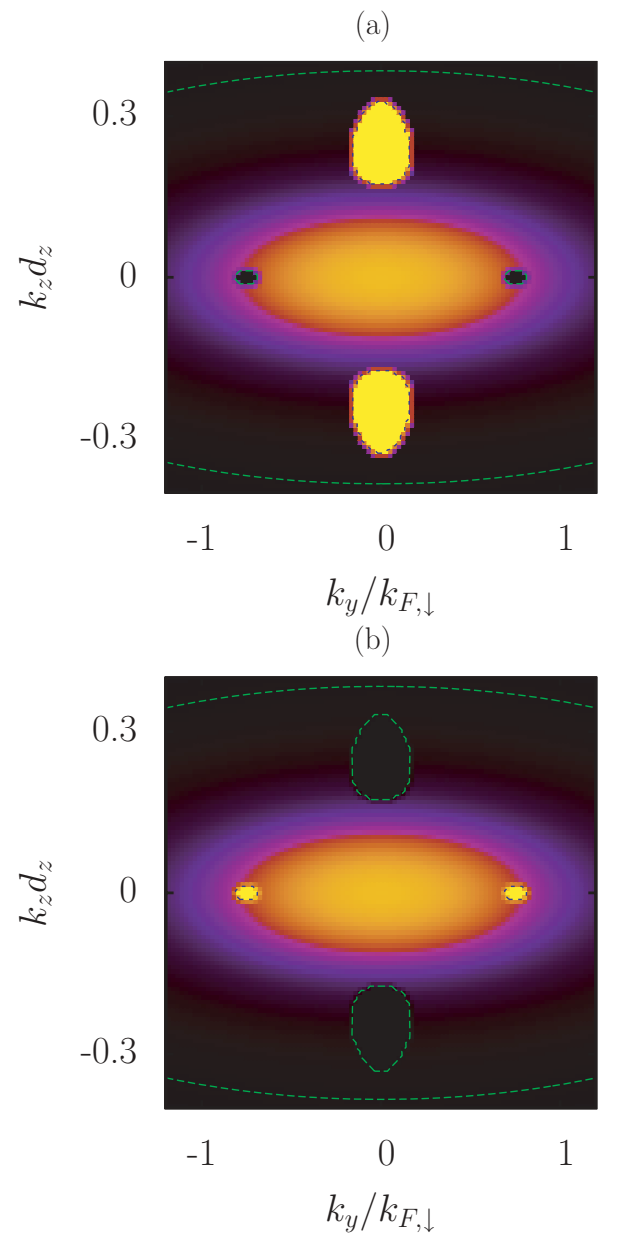

FIG. 3. (Color online) Contour maps of the momentum distributions (a) $n_{\mathbf{k}, \uparrow}$ for the $\uparrow$ fermions, and (b) $n_{\mathbf{k}, \downarrow}$ for the $\downarrow$ fermions, shown as a function of momentum components $k_{z}$ (in units of $1 / d_{z}$ ) and $k_{y}$ (in units of $\left.k_{F, \downarrow}\right)$ in the $k_{x}=0$ plane. These data correspond to the case considered in Fig. 2, where $m_{\uparrow}=m_{\downarrow}, t_{\uparrow}=\epsilon_{F, \downarrow}, k_{F, \downarrow} d_{z}=0.1$, and $1 /\left(k_{F, \downarrow} a_{\text {eff }}\right)=-0.25$. Note that when $E_{\mathbf{k}, 1}>0$ and $E_{\mathbf{k}, 2}>0$, then $n_{\mathbf{k}, \uparrow}=n_{\mathbf{k}, \downarrow}$. However, when $E_{\mathbf{k}, 1} \leqslant 0$ and $E_{\mathbf{k}, 2}>0$, then $n_{\mathbf{k}, \uparrow}=1$ and $n_{\mathbf{k}, \downarrow}=0$; and when $E_{\mathbf{k}, 1}>0$ and $E_{\mathbf{k}, 2} \leqslant 0$, then $n_{\mathbf{k}, \uparrow}=0$ and $n_{\mathbf{k}, \downarrow}=1$. The densities are 1 (0) in the bright yellow (black) regions.

indicated by an arrow. The excitations are gapless $\left(E_{\mathbf{k}, s} \leqslant 0\right)$ at the white region boundaries.

The topological transition could be potentially observed through the measurement of the momentum distribution $n_{\mathbf{k}, \sigma}$ of the fermions [27], which can be extracted from Eqs. (7) and (8). For k-space regions where $E_{\mathbf{k}, 1}>0$ and $E_{\mathbf{k}, 2}>0$, the corresponding momentum distributions are equal: $n_{\mathbf{k}, \uparrow}=$ $n_{\mathbf{k}, \downarrow}$. However, when $E_{\mathbf{k}, 1} \leqslant 0$ and $E_{\mathbf{k}, 2}>0$, then $n_{\mathbf{k}, \uparrow}=1$ and $n_{\mathbf{k}, \downarrow}=0$. Similarly, when $E_{\mathbf{k}, 1}>0$ and $E_{\mathbf{k}, 2} \leqslant 0$, then $n_{\mathbf{k}, \uparrow}=0$ and $n_{\mathbf{k}, \downarrow}=1$. We illustrate these cases in Fig. 3 for the parameters of Fig. 2. Note that, although there are excess (or unpaired) $\uparrow$ or $\downarrow$ fermions in different regions of the $\mathbf{k}$ space, i.e., the bright yellow regions, there are equal numbers of $\uparrow$ and $\downarrow$ fermions in total. The size of the yellow regions may look very different in (a) and (b) due partly to the difference in scaling factors in $k_{z}$ and $k_{y}$. 
This topological transition is quantum $(T=0)$ in nature, but its signatures should still be observed at finite temperatures within the quantum critical region, where the momentum distributions are smeared out due to thermal effects. In addition, while thermodynamic quantities such as atomic compressibility, specific heat, and spin susceptibility have power-law dependences on the temperature in the BCS side, they have exponential dependences on the temperature and the minimum energy of quasiparticle excitations in the BEC side, again signaling the existence of a quantum phase transition at $T=0$. Having discussed the topological classification of possible superfluid phases, we are ready to present the saddle-point phase diagrams at $T=0$, including the stability analysis (positive curvature criterion) of the solutions.

\section{Ground state phase diagrams}

In Fig. 4, ground state phase diagrams are shown as a function of the tunneling amplitude $t_{\uparrow}$ and the effective $s$-wave scattering parameter $1 /\left(k_{F, \downarrow} a_{\mathrm{eff}}\right)$ for fixed values of (a) $k_{F, \downarrow} d_{z}=0.1$ and (b) $k_{F, \downarrow} d_{z}=1$. We indicate normal (N), gapped uniform superfluid (SF), gapless uniform superfluid (gSF), and phase-separated (PS) regions. The normal phase is characterized by a vanishing order parameter $(\Delta=0)$, while the gapped superfluid and gapless superfluid phases are both characterized by $\Delta>0$ and $\partial^{2} \Omega_{0} / \partial \Delta^{2}>0$, but with distinct k-space topologies as discussed above. In the gSF phase, the unpaired $\uparrow$ and $\downarrow$ fermions coexist with the paired (or
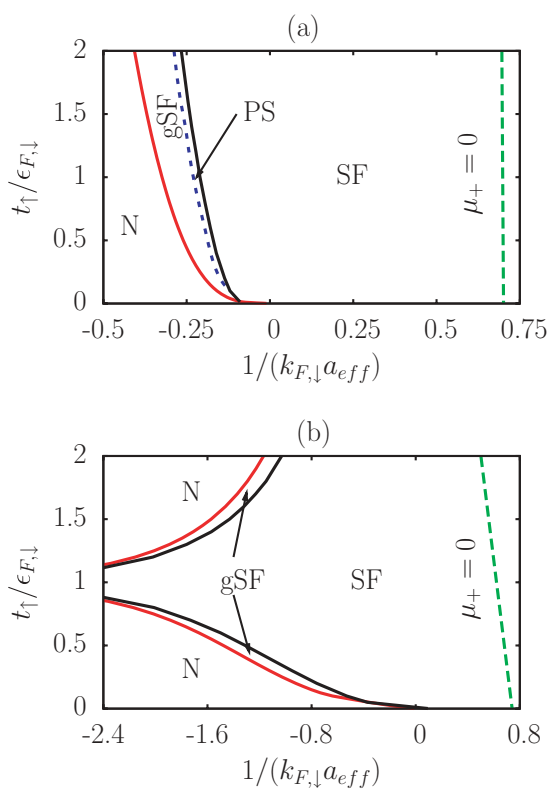

FIG. 4. (Color online) Ground state phase diagrams shown as a function of the tunneling amplitude $t_{\uparrow}$ (in units of $\epsilon_{F, \downarrow}$ ) and the effective $s$-wave scattering parameter $1 /\left(k_{F, \downarrow} a_{\text {eff }}\right)$. Here, we consider equal density $\left(n_{\uparrow}=n_{\downarrow}\right)$ and equal mass $\left(m_{\uparrow}=m_{\downarrow}\right)$ mixtures, for fixed values of $k_{F, \downarrow} d_{z}=0.1$ in (a) and $k_{F, \downarrow} d_{z}=1$ in (b). We show normal $(\mathrm{N})$, gapped uniform superfluid $(\mathrm{SF})$, gapless uniform superfluid (gSF), and phase-separated (PS) regions. The PS region in (b) is close to the normal-superfluid transition boundary, but it is very small and not shown. There is no phase transition across $\mu_{+}=0$ (green-dashed) line; it is shown only as a reference point. superfluid) ones in different $\mathbf{k}$-space regions, but there are no unpaired fermions in the SF phase, i.e., all $\uparrow$ and $\downarrow$ fermions are paired. The gSF phase is in some ways similar to the Sarma state found in mixtures with unequal densities [11], but in our case, the gapless superfluid phase is unpolarized and most importantly it is stable against phase separation. The phaseseparated region is characterized by $\partial^{2} \Omega_{0} / \partial \Delta^{2}<0$, but this region could also be of the Fulde-Ferrell-Larkin-Ovchinnikov (FFLO)-type superfluid having spatial modulations [28,29]. Such a possibility is not considered in this study, and it is left as an important problem to address in the future.

We can understand these phase diagrams as follows. For a fixed $t_{\uparrow}$, when the scattering parameter is smaller than a critical value, the potential energy is not sufficient to cause pairing due to mismatch of the Fermi surfaces, and the mixture is a normal Fermi gas with $\Delta=0$. As shown in Fig. 4(a) the critical scattering parameter decreases with increasing $t_{\uparrow}$, since increasing $t_{\uparrow}$ decreases the mismatch for lower values of $t_{\uparrow}$. In the normal region, when $t_{\uparrow}=0$, the Fermi surface of the $\uparrow$ fermions is a cylindrical shell in the $\mathbf{k}$ space with height $k_{F z, \uparrow}=2 \pi / d_{z}$ and radius $k_{F, \uparrow}$. However, in the low-filling limit of $\downarrow$ fermions, the Fermi surface of the $\downarrow$ fermions is more like a spherical shell with radius $k_{F, \downarrow}$. Note that for equal mass and equal density mixtures considered here, the k-space volumes enclosed by the cylindrical and spherical Fermi surfaces must be equal. Therefore, at $t_{\uparrow}=0$, there is a large mismatch between the two Fermi surfaces when $k_{F, \uparrow} \ll$ $k_{F, \downarrow} \ll \pi / d_{z}$, and increasing $t_{\uparrow}$ from zero decreases $k_{F z, \uparrow}$ and increases the ratio $k_{F, \uparrow} / k_{F, \downarrow}$. When this happens, the Fermi surface of the $\uparrow$ fermions looks like a prolate spheroid (like an American football). This decreases the mismatch for small values of $t_{\uparrow}$ as long as $k_{F, \uparrow} \lesssim k_{F, \downarrow}$, and it is qualitatively what happens along the normal-superfluid transition boundary in Fig. 4(a), when $k_{F, \downarrow} d_{z}=0.1 \ll \pi$.

In Fig. 4(b) we show the same phase diagram for a higher value of $k_{F, \downarrow} d_{z}=1$. Similar to Fig. 4(a) the critical scattering parameter for the normal-superfluid transition decreases initially with increasing $t_{\uparrow}$, since increasing $t_{\uparrow}$ decreases the mismatch for lower values of $t_{\uparrow}$. In contrast, beyond a critical value of $t_{\uparrow}$ [e.g., $t_{\uparrow} \gtrsim \epsilon_{F, \downarrow}$ in Fig. 4(b)] the critical scattering parameter increases with increasing $t_{\uparrow}$. This is because, since increasing $t_{\uparrow}$ decreases $k_{F, z}$ and increases the ratio $k_{F, \uparrow} / k_{F, \downarrow}$, it eventually increases the mismatch of the two Fermi surfaces after $k_{F, \uparrow}>k_{F, \downarrow}$. When this happens, the Fermi surface of the $\uparrow$ fermions changes from a prolate spheroid to an oblate spheroid (a disk-shaped ellipsoid). For the case considered in Fig. 4(a) where $k_{F, \downarrow} d_{z}=0.1$, we found that this occurs beyond $t_{\uparrow} \gtrsim 100 \epsilon_{F, \downarrow}$, but it is not shown. In general, for equal mass and equal density mixtures, such a change is expected to occur beyond $t_{\uparrow} \gtrsim 1 /\left(2 m_{\uparrow} d_{z}^{2}\right)$, and this expectation is consistent with our numerical findings.

In addition, in Fig. 4 the solid black lines correspond to the transition boundary between the gapless superfluid (gSF) and gapped superfluid (SF) phases. However, the stability criterion given in Eq. (9) is not satisfied in some parts of the gapless superfluid region, indicating a phase separation. This occurs between the dashed-blue and the solid-black lines in Fig. 4(a) In contrast, phase separation occurs in a tiny region very close to the normal-superfluid transition boundary (solid red line) in Fig. 4(b) and it is not shown. The gapless superfluid phase 
phase is in some ways similar to the Sarma state found in mixtures with unequal densities [11], but in our case, gSF is unpolarized and most importantly it is stable against phase separation in a considerably large region as shown in the figures. In this way, our gSF phase is similar to those of Refs. [12,13], which are recently proposed for ultracold atomic systems in other contexts.

Before concluding, we would like to comment on the phase diagram of mixed-dimensional two-species FermiFermi mixtures. When $\uparrow$ and $\downarrow$ fermions have different masses, the phase boundaries shift left (right) when the $\uparrow$ species is heavier (lighter) than the $\downarrow$ fermions. For instance, in the case of ${ }^{6} \mathrm{Li}^{4}{ }^{40} \mathrm{~K}$ mixtures, the $t_{\uparrow} \rightarrow 0$ limit of the normalsuperfluid boundary for the case considered in Fig. 4(a) shifts to $1 /\left(k_{F, \downarrow} a_{\text {eff }}\right) \approx-0.6$ when $m_{\uparrow}=6.64 m_{\downarrow}$ (when ${ }^{40} \mathrm{~K}$ atoms correspond to $\uparrow)$ and to $1 /\left(k_{F, \downarrow} a_{\mathrm{eff}}\right) \approx 0.6$ when $m_{\uparrow}=0.15 m_{\downarrow}$ (when ${ }^{6} \mathrm{Li}$-atoms correspond to $\uparrow$ ).

\section{CONCLUSIONS}

Motivated by a very recent experiment involving mixeddimensional Bose-Bose mixtures [10], here we investigated the ground state phase diagram of superfluidity for mixeddimensional Fermi-Fermi mixtures in the BCS-BEC evolution. In this recent experiment, a species-selective one-dimensional optical lattice was applied to a two-species mixture of bosonic atoms, such that only one of the species feels the lattice potential and is confined to a quasi-two-dimensional geometry, while having negligible effect on the other, thus leaving it three dimensional. We considered a similar problem with two-species mixtures of fermionic atoms, where both species are confined to quasi-two-dimensional geometries determined by their hoppings along the lattice direction.

We considered equal-density mixtures at zero temperature, and after solving the saddle-point self-consistency equations, we constructed the phase diagrams using some stability criterion. We found normal, gapped superfluid, gapless superfluid, and phase separated regions. The gapped superfluid and gapless superfluid phases are identified with the disappearance or appearance of momentum space regions of zero quasiparticle energies. In particular, we found a stable gapless superfluid phase where the unpaired $\uparrow$ and $\downarrow$ fermions coexist with the paired (or superfluid) ones in different momentum space regions. This phase is in some ways similar to the Sarma state found in mixtures with unequal densities [11], but in our case, the gapless superfluid phase is unpolarized and most importantly it is stable against phase separation. We also argued that the topological transition from the gapped superfluid to the gapless superfluid could be potentially observed through the measurement of the momentum distribution [27].

There are several ways to extend this work. First, the possibility of FFLO-type superfluid phases [28,29], where Cooper pairs have finite center of mass momentum, leading to a spatially modulated phase, is not considered in this study. This is an important problem to address due to its relevance to condensed-matter systems. Second, our calculation is based on the saddle-point self-consistency equations, which are known to be sufficient to qualitatively describe the entire BCS-BEC evolution, at least for the usual three-dimensional mixtures at low temperatures. However, corrections beyond the saddle point could be important in stabilizing or destablizing especially the gapless superfluid phase [30]. Third, we used a single-band model to describe the optical lattice potential, and the effects of higher bands could become important near the strongly interacting regime. Lastly, atomic systems are not uniform since confining trapping potentials are always present, and finite-size effects due to such potentials could also be analyzed.

\section{ACKNOWLEDGMENTS}

We thank the Scientific and Technological Research Council of Turkey (TÜBITAK) for financial support, and Institute of Theoretical and Applied Physics (ITAP - Marmaris) for their hospitality. M.I. is also supported by a Marie Curie International Reintegration Grant (FP7-PEOPLE-IRG-2010268239).

\section{APPENDIX A: SELF-CONSISTENCY EQUATIONS AT $T=0$}

For numerical purposes, the self-consistency equations can be simplified as follows. At zero temperature, since the Fermi function $f(x)$ turns into a Heaviside step function $\theta(-x)$, Eqs. (6), (7), and (8) become

$$
\begin{gathered}
\frac{m_{+} V}{4 \pi a_{\mathrm{eff}}}=\sum_{\mathbf{k}}\left[\frac{1}{2 \epsilon_{\mathbf{k},+}}-\frac{1-\theta\left(-E_{\mathbf{k}, 1}\right)-\theta\left(-E_{\mathbf{k}, 2}\right)}{2 E_{\mathbf{k},+}}\right], \\
N_{\uparrow}=\sum_{\mathbf{k}}\left[\left|u_{\mathbf{k}}\right|^{2} \theta\left(-E_{\mathbf{k}, 1}\right)+\left|v_{\mathbf{k}}\right|^{2} \theta\left(E_{\mathbf{k}, 2}\right)\right], \\
N_{\downarrow}=\sum_{\mathbf{k}}\left[\left|u_{\mathbf{k}}\right|^{2} \theta\left(-E_{\mathbf{k}, 2}\right)+\left|v_{\mathbf{k}}\right|^{2} \theta\left(E_{\mathbf{k}, 1}\right)\right],
\end{gathered}
$$

where the $\mathbf{k}$-space sums are $\sum_{\mathbf{k}} \equiv\left[V /(2 \pi)^{3}\right] \int d^{3} \mathbf{k} \equiv$ $\left[V /\left(2 \pi^{2}\right)\right] \int_{0}^{\pi / d_{z}} d k_{z} \int_{0}^{\infty} k_{\perp} d k_{\perp}$. Note that pairing occurs only in the k-space regions where both $E_{\mathbf{k}, 1}$ and $E_{\mathbf{k}, 2}$ have the same (positive) sign. When $E_{\mathbf{k}, 1} \leqslant 0$ and $E_{\mathbf{k}, 2} \geqslant 0$ or vice versa, the second term (on the right-hand side) of Eq. (A1) inside the parentheses vanishes, reflecting that the pairing is not allowed for those k-space regions, and the quasiparticle and quasihole excitations are gapless.

In order to perform the integration over $k_{\perp}$ by hand, we need to find the $\mathbf{k}$-space regions where the excitations are gapless, i.e., $E_{\mathbf{k}, 1} \leqslant 0$ or $E_{\mathbf{k}, 2} \leqslant 0$. The zeros of $E_{\mathbf{k}, 1}$ and $E_{\mathbf{k}, 2}$ are determined by real and positive solutions of

$$
\begin{aligned}
0= & \left(1-m_{+}^{2} / m_{-}^{2}\right) x^{2}+2\left(\xi_{k_{z},+}-m_{+} \xi_{k_{z},-} / m_{-}\right) x \\
& +\xi_{k_{z}, \uparrow} \xi_{k_{z}, \downarrow}+|\Delta|^{2},
\end{aligned}
$$

where $x=k_{\perp}^{2} /\left(2 m_{+}\right)$and $\xi_{k_{z}, \pm}=\epsilon_{k_{z}, \pm}-\mu_{ \pm}$. Here, we introduced $\epsilon_{k_{z}, \pm}=\left(\epsilon_{k_{z}, \uparrow} \pm \epsilon_{k_{z}, \downarrow}\right) / 2$, where $\epsilon_{k_{z}, \sigma}=2 t_{\sigma}[1-$ $\left.\cos \left(k_{z} d_{z}\right)\right]$ is the energy dispersion in the $k_{z}$ direction. Solutions of this equation $\left(x_{<}\right.$and $\left.x_{>}\right)$depend on $k_{z}$, and they give the locations of the zeros of $E_{\mathbf{k}, 1}$ and $E_{\mathbf{k}, 2}$ in the $k_{\perp}$-axis as a function of $k_{z}$. For instance, if both $x_{<}$and $x_{>}$are real and positive, then $E_{\mathbf{k}, 1}$ is gapless for $x_{<} \leqslant k_{\perp}^{2} /\left(2 m_{+}\right) \leqslant x_{>}$ in some $k_{z}$ region $z_{<, 1} \leqslant k_{z} \leqslant z_{>, 1}$, and $E_{\mathbf{k}, 2}$ is gapless for $x_{<} \leqslant k_{\perp}^{2} /\left(2 m_{+}\right) \leqslant x_{>}$in some other $k_{z}$ region $z_{<, 2} \leqslant k_{z} \leqslant$ $z_{>}$. If only $x_{>}$is real and positive, $E_{\mathbf{k}, 1}$ is gapless for $0 \leqslant k_{\perp}^{2} /\left(2 m_{+}\right) \leqslant x_{>}$in some $k_{z}$ region $z_{<, 1} \leqslant k_{z} \leqslant z_{>, 1}$, and $E_{\mathbf{k}, 2}$ is gapless for $0 \leqslant k_{\perp}^{2} /\left(2 m_{+}\right) \leqslant x_{>}$in some other $k_{z}$ region 
$z_{<, 2} \leqslant k_{z} \leqslant z_{>, 2}$. If there is no real and positive solution, then the excitations are always gapped.
Given the gapless k-space regions, Eq. (A1) can be written as

$$
\frac{\pi}{a_{\mathrm{eff}}}=\left(\int_{z_{<, 1}}^{z_{>, 1}}+\int_{z_{<, 2}}^{z_{>, 2}}\right) d k_{z} \ln \left[\frac{x_{>}+\xi_{k_{z},+}+\sqrt{\left(x_{>}+\xi_{k_{z},+}\right)^{2}+|\Delta|^{2}}}{x_{<}+\xi_{k_{z},+}+\sqrt{\left(x_{<}+\xi_{k_{z},+}\right)^{2}+|\Delta|^{2}}}\right]-\int_{0}^{\pi / d_{z}} d k_{z} \ln \left(\frac{2 \epsilon_{k_{z},+}}{\xi_{k_{z},+}+\sqrt{\xi_{k_{z},+}^{2}+|\Delta|^{2}}}\right),
$$

where the first term on the right-hand side is coming from the gapless, but the second term is from the gapped k-space regions. Similarly, Eq. (A2) can be written as

$$
\begin{aligned}
\frac{4 \pi^{2} n_{\uparrow}}{m_{+}}= & -\left(\int_{z_{<, 1}}^{z_{>, 1}}+\int_{z_{<, 2}}^{z_{>, 2}}\right) d k_{z}\left[x_{>}-\sqrt{\left(x_{>}+\xi_{k_{z},+}\right)^{2}+|\Delta|^{2}}-x_{<}+\sqrt{\left(x_{<}+\xi_{k_{z},+}\right)^{2}+|\Delta|^{2}}\right] \\
& +2 \int_{z_{<, 1}}^{z_{>, 1}} d k_{z}\left(x_{>}-x_{<}\right)+\int_{0}^{\pi / d_{z}} d k_{z}\left(\sqrt{\xi_{k_{z},+}^{2}+|\Delta|^{2}}-\xi_{k_{z},+}\right)
\end{aligned}
$$

where again the first two terms on the right-hand side are coming from the gapless, but the third term is from the gapped k-space regions. The density of $\downarrow$ fermions can be obtained by substituting $1 \rightarrow 2$ in the integration limits of the second term. Our numerical calculations show that integrating $k_{\perp}$ by hand as described above and calculating the remaining $k_{z}$ integral numerically is a much more stable approach compared to the one where both integrations are calculated numerically. In particular, this approach converges much faster than the latter on the BCS side, where integrations involve gapless k-space regions.

\section{APPENDIX B: NORMAL-SUPERFLUID PHASE BOUNDARY AT $T=0$}

The phase boundary for the normal-superfluid transition can be found from Eqs. (6), (7) and (8) by setting $\Delta=0$. Therefore, at the transition boundary, the self-consistency equations are uncoupled, i.e., the gap equation determines the critical effective scattering length, and the chemical potentials are determined by the number equations. At zero temperature, this leads to

$$
\frac{m_{+} V}{4 \pi a_{\mathrm{eff}}}=\sum_{\mathbf{k}}\left[\frac{1}{2 \epsilon_{\mathbf{k},+}}-\frac{1-\theta\left(-\xi_{\mathbf{k}, \uparrow}\right)-\theta\left(-\xi_{\mathbf{k}, \downarrow}\right)}{2 \xi_{\mathbf{k},+}}\right]
$$

$$
N_{\sigma}=\sum_{\mathbf{k}} \theta\left(-\xi_{\mathbf{k}, \sigma}\right) .
$$

Note that Eq. (A2) is the number equation for noninteracting fermions, and it has already been solved in Sec. II C. Similar to Appendix A, we can also simplify Eq. (6) by finding the zeros of $\xi_{\mathbf{k}, \sigma}$. This leads to

$$
\begin{aligned}
-\frac{\pi}{a_{\mathrm{eff}}}= & \int_{0}^{k_{F z,<}} d k_{z} \ln \left(\frac{\xi_{k_{z},+}}{\xi_{k_{z},+}-\frac{m_{<}}{m_{+}} \xi_{k_{z},<}}\right) \\
& +\int_{0}^{k_{F z,>}} d k_{z} \ln \left(\frac{\epsilon_{k_{z},+}}{\xi_{k_{z},+}-\frac{m_{>}}{m_{+}} \xi_{k_{z},>}}\right) \\
& +\int_{k_{F z,>}}^{\pi / d_{z}} d k_{z} \ln \left(\frac{\epsilon_{k_{z},+}}{\xi_{k_{z},+}}\right)
\end{aligned}
$$

where $k_{F z,<} \equiv \min \left\{k_{F z, \uparrow}, k_{F z, \downarrow}\right\} \quad$ and $k_{F z,>} \equiv \max \left\{k_{F z, \uparrow}\right.$, $\left.k_{F z, \downarrow}\right\}$. For instance, when $k_{F z,<} \equiv k_{F z, \downarrow}$, then $<\equiv \downarrow$ and $>\equiv \uparrow$ in the integrands. Here, we again emphasize that integrating $k_{\perp}$ by hand as described above and calculating the remaining $k_{z}$ integral numerically are a much more stable approach compared to the one where both integrations are calculated numerically.
[1] M. Inguscio, W. Ketterle, and C. Salomon, Ultra-cold Fermi Gases, Proceedings of the International School of Physics Enrico Fermi, Course CLXIV, Varenna (2006).

[2] M. Taglieber, A.-C. Voigt, T. Aoki, T. W. Hänsch, and K. Dieckmann, Phys. Rev. Lett. 100, 010401 (2008).

[3] E. Wille et al., Phys. Rev. Lett. 100, 053201 (2008).

[4] A.-C. Voigt, M. Taglieber, L. Costa, T. Aoki, W. Wieser, T. W. Hänsch, and K. Dieckmann, Phys. Rev. Lett. 102, 020405 (2009).

[5] F. M. Spiegelhalder, A. Trenkwalder, D. Naik, G. Hendl, F. Schreck, and R. Grimm, Phys. Rev. Lett. 103, 223203 (2009).
[6] T. G. Tiecke, M. R. Goosen, A. Ludewig, S. D. Gensemer, S. Kraft, S. J. J. M. F. Kokkelmans, and J. T. M. Walraven, Phys. Rev. Lett. 104, 053202 (2010).

[7] F. M. Spiegelhalder, A. Trenkwalder, D. Naik, G. Kerner, E. Wille, G. Hendl, F. Schreck, and R. Grimm, Phys. Rev. A 81, 043637 (2010).

[8] Y. Nishida and S. Tan, Phys. Rev. Lett. 101, 170401 (2008).

[9] Y. Nishida, Phys. Rev. A 82, 011605(R) (2010).

[10] G. Lamporesi, J. Catani, G. Barontini, Y. Nishida, M. Inguscio, and F. Minardi, Phys. Rev. Lett. 104, 153202 (2010).

[11] G. Sarma, J. Phys. Chem. Solids 24, 1029 (1963).

[12] M. Iskin and E. Tiesinga, Phys. Rev. A 79, 053621 (2009). 
[13] A. E. Feiguin and M. P. A. Fisher, Phys. Rev. Lett. 103, 025303 (2009).

[14] M. W. Zwierlein et al., Science 311, 492 (2006).

[15] G. B. Partridge et al., Science 311, 503 (2006).

[16] Y. Shin et al., Nature (London) 451, 689 (2008).

[17] N. Navon et al., Science 328, 729 (2010).

[18] Rigorously speaking one has to take into account all the momentum states beyond the first Brillouin zone; however, almost all "high-momentum" effects are absorbed in the effective $s$-wave scattering parameter $a_{\text {eff }}$, and this is hopefully sufficient for the qualitative description of the problem. Note that the relation between the three-dimensional scattering length $a_{s}$ and $a_{\text {eff }}$ can be established by solving the two-body problem, and that infinite $a_{s}$ does not mean infinite $a_{\mathrm{eff}}$ in general.

[19] A. J. Leggett, Modern Trends in the Theory of Condensed Matter (Springer-Verlag, Berlin, 1980), p. 13.

[20] J. R. Engelbrecht, M. Randeria, and C. A. R. Sa de Melo, Phys. Rev. B 55, 15153 (1997).

[21] M. Iskin and C. A. R. Sá de Melo, Phys. Rev. Lett. 97, 100404 (2006).

[22] L. He, M. Jin, and P. Zhuang, Phys. Rev. B 74, 214516 (2006).
[23] C.-H. Pao, S.-T. Wu, and S.-K. Yip, e-print arXiv:cond-mat/0608501.

[24] A. M. Clogston, Phys. Rev. Lett. 9, 266 (1962).

[25] B. S. Chandrasekhar, Appl. Phys. Lett. 1, 7 (1962).

[26] G. E. Volovik, Lect. Notes Phys. 718, 31 (2007); see also e-print arXiv:cond-mat/0601372.

[27] In cold-atom experiments, the peculiar momentum distribution of different topological phases would be smeared out by the trapping potential, but their marked signatures should still be present.

[28] P. Fulde and R. A. Ferrell, Phys. Rev. 135, A550 (1964).

[29] A. I. Larkin and Y. N. Ovchinnikov, Sov. Phys. JETP 20, 762 (1965).

[30] In mixed-dimensional systems, it is not obvious whether the saddle-point equations are sufficient to describe the BCS-BEC evolution of superfluidity or not. For instance, in a bilayer system [9], it has been proposed that the normal phase in the weak coupling region becomes an interlayer $s$-wave or intralayer $p$-wave superfluid phase depending on the layer separation $d_{z}$. In addition, it is also possible that two two-dimensional atoms in different layers and one three-dimensional atom can form a trimer state whose binding energy scales as $1 / d_{z}^{2}$. 